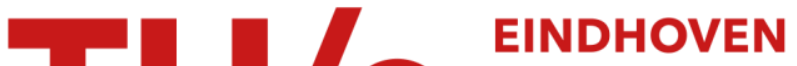 UNIVERSITY OF TECHNOLOGY
}

\section{A design approach for noncausal robust iterative learning control using worst case disturbance optimisation}

\section{Citation for published version (APA):}

Donkers, M. C. F., Wijdeven, van de, J. J. M., \& Bosgra, O. H. (2008). A design approach for noncausal robust iterative learning control using worst case disturbance optimisation. In Proceedings of the 2008 American Control Conference (ACC2008), Seattle, Washington, USA, June 11-13, 2008 (pp. 4567-4572). Institute of Electrical and Electronics Engineers. https://doi.org/10.1109/ACC.2008.4587215

DOI:

10.1109/ACC. 2008.4587215

Document status and date:

Published: 01/01/2008

\section{Document Version:}

Publisher's PDF, also known as Version of Record (includes final page, issue and volume numbers)

\section{Please check the document version of this publication:}

- A submitted manuscript is the version of the article upon submission and before peer-review. There can be important differences between the submitted version and the official published version of record. People interested in the research are advised to contact the author for the final version of the publication, or visit the $\mathrm{DOI}$ to the publisher's website.

- The final author version and the galley proof are versions of the publication after peer review.

- The final published version features the final layout of the paper including the volume, issue and page numbers.

Link to publication

\section{General rights}

Copyright and moral rights for the publications made accessible in the public portal are retained by the authors and/or other copyright owners and it is a condition of accessing publications that users recognise and abide by the legal requirements associated with these rights.

- Users may download and print one copy of any publication from the public portal for the purpose of private study or research.

- You may not further distribute the material or use it for any profit-making activity or commercial gain

- You may freely distribute the URL identifying the publication in the public portal.

If the publication is distributed under the terms of Article 25fa of the Dutch Copyright Act, indicated by the "Taverne" license above, please follow below link for the End User Agreement:

www.tue.nl/taverne

Take down policy

If you believe that this document breaches copyright please contact us at:

openaccess@tue.nl

providing details and we will investigate your claim. 


\title{
A Design Approach for Noncausal Robust Iterative Learning Control using Worst Case Disturbance Optimisation
}

\author{
Tijs Donkers, Jeroen van de Wijdeven, Okko Bosgra \\ Department of Mechanical Engineering \\ Eindhoven University of Technology \\ P.O.Box 513, 5600 MB Eindhoven, The Netherlands \\ \{m.c.f.donkers,j.j.m.v.d.wijdeven, o.h.bosgra\}@tue.nl
}

\begin{abstract}
In this paper, we present a novel Iterative Learning Control (ILC) strategy that is robust against model uncertainty, as given by a system model and an additive uncertainty bound. The design methodology hinges on $\mathcal{H}_{\infty}$ optimisation, however, the procedure is modified such that the ILC controller is noncausal and inherently acts on a finite time interval. The resulting controller has the structure of a norm optimal ILC controller, so that robustness can be easily assessed. Furthermore, in an example, we show that the presented robust ILC controller can outperform linear quadratic ILC controllers.
\end{abstract}

\section{INTRODUCTION}

Iterative Learning Control (ILC) is a control strategy that can be applied to high performance systems that perform a task repeatedly. Since the task is repetitive, it sounds natural to include experience from previous trials to improve performance of the controlled system in the subsequent trial. Hence, by learning from previous errors. A properly designed ILC controller iteratively finds a command signal that yields high system performance. For an introduction to ILC, the reader is referred to [7].

Although the command signal generated by the ILC controller is based on measured data, the controller is designed using a system model. Since no model can truly reflect the real system behaviour, the controller is required to have some robustness against trial invariant model uncertainty. Depending on the amount of uncertainty present, and on the robustness of the controller itself, the ILC controlled system can become unstable, rendering ILC useless.

A number of contributions have been made that study the robustness of ILC against model uncertainty, i.e., Robust ILC (R-ILC). For a norm optimal ILC controller (see, e.g., [3], [10], [14]), it is recognised that it has some robustness against model uncertainty. To quantify the allowable uncertainty, tools have been developed in [9], [11], [13]. Although an uncertainty model is used to analyse robustness, the ILC controller itself does not incorporate such an uncertainty model, resulting in a declined performance of the ILC algorithm.

A class of R-ILC controllers that do incorporate an uncertainty model in the design of a controller, pose the design problem as an $\mathcal{H}_{\infty}$ optimisation problem, [4], [19]. Herein, the design problem is posed in the frequency domain, and therefore, yields an approximate result. This is due to the fact that the Fourier transform assumes that signals act on an infinite time interval, whereas in ILC, they inherently act on a finite time interval. Moreover, the resulting $\mathcal{H}_{\infty}$ optimal controllers are causal, which is also a limitation. Causality of ILC controllers refers to the fact that the command signal in trial $k+1$ at time $t$ only depends on information of trial $k$ at time $[0, \ldots, t-1]$. Though, according to [12], [21], the real benefit of ILC lies in the noncausality of the solution. In [16], the ILC controller problem is formulated as an $\mathcal{H}_{\infty}$ problem in the trial domain, but trial varying uncertainty is discussed, instead of trial invariant uncertainty.

Another suggestion that uses an uncertainty model for designing an ILC controller, is made in [1], [2]. Herein, model uncertainty is represented as interval uncertainty in the system's impulse response. Although the resulting controllers are noncausal and inherently act on a finite time interval, synthesis of these controllers can be numerically demanding.

In this paper, we present an R-ILC controller, with a structure similar to that of norm optimal ILC controllers, that incorporates an uncertainty model in the controller. Because of this similar structure, we can use results of [9] to show that the ILC algorithm is robust. For the derivation of the controller, we use a procedure similar to $\mathcal{H}_{\infty}$ optimisation, however, modified in such a way that the solution becomes noncausal and inherently acts on a finite time interval. A similar procedure is presented in [22], however, in this paper we can make statements about robustness in a more elegant framework.

The remainder of this paper is organised as follows. In Section II, we introduce the necessary ILC notations. Subsequently, in Section III, we quickly review the ideas and results of [9], by defining the robust monotonic convergence problem and by giving sufficient conditions for robust monotonic convergence. The main contribution of this paper, the R-ILC solution, is presented in Section IV. In Section V, a simulation example is discussed that shows that the presented R-ILC controller outperforms the conventional norm optimal ILC controller, while retaining its robustness. Finally, some conclusions are drawn in Section VI.

\section{NOMENCLATURE}

In this paper, we consider discrete time, Linear Time Invariant (LTI) systems, with $l$ outputs and $m$ inputs. Since 
for these systems the $z$-transform exists, we can represent a set of perturbed systems $\Pi_{z}$ with a bounded additive uncertainty as follows:

$\Pi_{z}:\left\{J_{p}(z)=J(z)+W_{i}(z) \Delta(z) W_{o}(z):\|\Delta(z)\|_{i 2} \leq 1\right\}$.

In (1), $J(z)$ represents the nominal model, $W_{i}(z)$ and $W_{o}(z)$ form a bound on the additive uncertainty, and $\Delta(z)$ is an arbitrary, stable system.

Since ILC explicitly acts on a finite time interval $t \in[0,1, \ldots, N-1]$, we can use the lifted setting, as first introduced in [18], to express our systems and filters. In this setting, every time signal in trial $k$ is stored in either an $l N$ - or an $m N$-dimensional column vector, e.g.:

$$
y_{k}=\left[y_{k}^{T}(0), \quad y_{k}^{T}\left(T_{s}\right), \quad \ldots, \quad y_{k}^{T}\left((N-1) T_{s}\right)\right]^{T},
$$

where $T_{s}$ denotes the sampling time. For brevity of notation, $T_{s}$ is omitted in the remainder of this paper. In the same setting, systems are represented by its convolution matrix:

$$
J=\left[\begin{array}{cccc}
j(0) & 0 & \ldots & 0 \\
j(1) & j(0) & & \vdots \\
\vdots & \ddots & \ddots & 0 \\
j(N-1) & \cdots & j(1) & j(0)
\end{array}\right]
$$

where the sequence $\{j(0), j(1), \ldots, j(N-1)\}$, with $j(t) \in$ $\mathbb{R}^{l \times m}$, denotes the system's Markov parameters. The Markov parameters result from observing the system's response to a unit pulse. The matrices $W_{i}$ and $W_{o}$ are derived from $W_{i}(z)$ and $W_{o}(z)$, respectively, similar as $J$ from $J(z)$. Using the lifted notation, a finite time representation of (1) can be written as:

$$
\Pi:\left\{J_{p}=J+W_{i} \Delta W_{o}:\|\Delta\|_{i 2} \leq 1\right\} .
$$

The set $\Pi$ now maps an input vector $f_{k} \in \mathbb{R}^{m N}$ to an output vector $y_{k} \in \mathbb{R}^{l N}$, i.e., $y_{k}=J_{p} f_{k}$. The lifted system $\Delta$ of (4) represents an arbitrary, norm bounded, lower triangular, block Toeplitz matrix. Although, representing uncertainty as in (4) may be a novel idea for ILC, it is a mature concept in the field of robust control theory (see, e.g., [20]).

In this paper, we use both the $z$-domain and the lifted description. To avoid any confusion, all $z$-domain signals and systems will have the index $z$.

Furthermore, in this paper, we make extensive use of norms. Given a lifted description, the induced 2-norm is defined as follows:

$$
\|J\|_{i 2}=\sup _{f \neq 0} \frac{\|J f\|_{2}}{\|f\|_{2}}=\bar{\sigma}(J),
$$

where $\|f\|_{2}=\sqrt{\langle f, f\rangle}$ denotes the 2-norm for vectors and $\bar{\sigma}$ denotes the maximum singular value.

\section{CONDitions For Robust MonotoniC CONVERGENCE}

In this Section, we revise the results presented in [9], where the notion of robust monotonic convergence (RMC) is formulated and conditions for RMC for norm optimal ILC

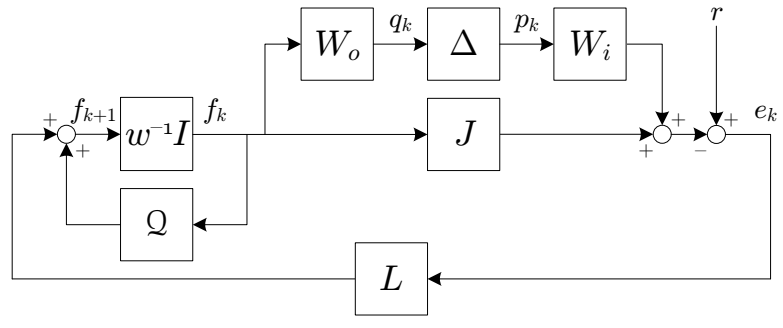

Fig. 1: General ILC control structure.

controllers are derived. Yet first, let us consider the ILC control structure used in this paper. This control structure is similar to the one used in [23] and is shown in Fig. 1. The corresponding trial domain dynamics are:

$$
\left\{\begin{array}{l}
f_{k+1}=\mathcal{Q} f_{k}+L e_{k} \\
e_{k}=r-J_{p} f_{k},
\end{array}\right.
$$

with the corresponding closed loop dynamics:

$$
f_{k+1}=\left(\mathcal{Q}-L J_{p}\right) f_{k}+L r .
$$

In [7], [17], conditions for stability and convergence of the ILC controlled system are given. We extend the notion of monotonic convergence to include model uncertainty.

Definition 3.1 (Robust Monotonic Convergence): Given a $\mathcal{Q}$ and $L$, the ILC system (7) has the property Robust Monotonic Convergence (RMC) if there exists an $0 \leq \alpha<1$ such that for all $J_{p} \in \Pi$ :

$$
\left\|f_{k+1}-f_{\infty}\right\|_{2} \leq \alpha\left\|f_{k}-f_{\infty}\right\|_{2}
$$

with:

$$
\alpha=\left\|\mathcal{Q}-L J_{p}\right\|_{i 2},
$$

and $f_{\infty}=\lim _{k \rightarrow \infty} f_{k}$.

The difference between monotonic convergence and RMC is that in the former case we only guarantee the command signal to converge monotonically for $J_{p}=J$.

In [9], conditions for RMC are derived for norm optimal ILC controllers. These controllers have the following filters:

$$
\begin{aligned}
\mathcal{Q} & =\left(J^{T} Q J+R+S\right)^{-1}\left(J^{T} Q J+R\right), \\
L & =\left(J^{T} Q J+R+S\right)^{-1} J^{T} Q,
\end{aligned}
$$

where $Q=Q^{T}>0, R=R^{T} \geq 0$, and $S=S^{T} \geq 0$ denote weighting matrices. Note the difference between $\mathcal{Q}$ and $Q$ : the former is a filter, while the latter is a weighting matrix.

In [9], [13] it was proved that allowable model uncertainty is not influenced by $R$. Considering this fact, sufficient conditions for RMC are given in the following Proposition [9].

Proposition 3.1: Given system (7), with $\mathcal{Q}$ and $L$ given by (10a) and (10b), respectively. Then, for MIMO systems (4) the ILC algorithm is RMC for any $R=\rho I \geq 0$, if:

$$
\left\|W_{o}\right\|_{i 2} \cdot\left\|\left(J^{T} Q J+S\right)^{-1} J^{T} Q W_{i}\right\|_{i 2}<1 .
$$


Furthermore, for SISO systems (4) and $W_{o}$ square, the ILC algorithm is RMC if:

$$
\left\|\left(J^{T} Q J+S\right)^{-1} J^{T} Q W_{i} W_{o}\right\|_{i 2}<1 .
$$

\section{R-ILC and the Minimax Game}

Using the results of the previous Section, it has become possible to design a norm optimal ILC controller that has RMC. As the main contribution of this paper, we discuss a procedure that results in an R-ILC controller that explicitly incorporates an uncertainty model. As it turns out, the structure of the controller is similar to that of norm optimal ILC controllers. For this, we present a theory similar to discrete time $\mathcal{H}_{\infty}$ control theory [5], [15], but modified in such a way that the solution is noncausal and inherently finite time. Hence, since the Hardy space refers to a class of stable, causal transfer functions, the name $\mathcal{H}_{\infty}$ is not appropriate for this solution.

\section{A. General Formulation}

A common approach in robust control theory is to formulate the problem using the generalised plant paradigm, that is depicted in Fig. 2. Given a generalised plant $P$, the control problem is to find a controller $K$ that minimises the performance outputs $z$, which are disturbed by disturbances $w$, using controlled inputs $u$ and measured outputs $y$.

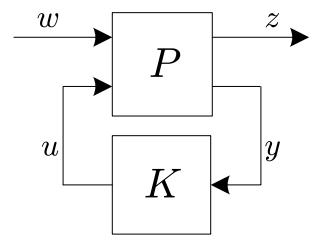

Fig. 2: The generalised plant paradigm.

In [5], [15], finite time $\mathcal{H}_{\infty}$ is discussed. A suboptimal controller is found by solving the following minimax criterion:

$$
\min _{u} \max _{w} \mathcal{J}(w(t), u(t), z(t), y(t)),
$$

where:

$$
\mathcal{J}=\sum_{t=0}^{N-1} z^{T}(t) z(t)-\gamma^{2} w^{T}(t) w(t),
$$

Using an observation made in [3], this cost functional can be converted into a lifted domain cost functional:

$$
\mathcal{J}=z^{T} z-\gamma^{2} w^{T} w .
$$

Optimal control problems are usually posed as constrained optimisation problems. The constraints stem from the system dynamics and from relations describing measured outputs. Since in the lifted domain, the system dynamics are hidden inside the Toeplitz matrices, the constraints consist of the measured output relations only. These constraints can be added to (15) using Lagrange multipliers (see, e.g., [6]).
Then, the solution that minimises the maximum disturbance is found where the constrained cost functional has a saddle point, i.e., where the Jacobian of the cost functional equals zero.

\section{B. R-ILC using a Generalised Plant Formulation}

The objective of an ILC controller is to minimise the error $e_{k+1}$ using measured information of $e_{k}$ and $f_{k}$. According to Fig. 1, the error at trial $k$ is as follows:

$$
\left\{\begin{array}{l}
e_{k}=r-J f_{k}-W_{i} p_{k} \\
q_{k}=W_{o} f_{k},
\end{array}\right.
$$

Since the reference trajectory $r$ is equal for each trial, the error at trial $k+1$ can be described as follows:

$$
\begin{cases}e_{k+1} & =e_{k}+J f_{k}+W_{i} p_{k}-J f_{k+1}-W_{i} p_{k+1} \\ q_{k} & =W_{o} f_{k} \\ q_{k+1} & =W_{o} f_{k+1} .\end{cases}
$$

Furthermore, in norm optimal ILC, it is common to limit the change of command signal between two subsequent trials, i.e., by weighting $f_{\Delta}=f_{k+1}-f_{k}$. We can add this requirement to the generalised plant, using weighting matrix $R^{1 / 2}=\sqrt{\rho} I$, such that $R=R^{1 / 2} R^{1 / 2}$. Using the fact that $e_{k}$ and $f_{k}$ are measured outputs, we can define the inputs and outputs of the generalised plant as follows:

$$
\begin{aligned}
w & =\left[\begin{array}{llll}
p_{k}^{T} & p_{k+1}^{T} & e_{k}^{T} & f_{k}^{T}
\end{array}\right]^{T} \\
u & =f_{k+1} \\
z & =\left[\begin{array}{llll}
q_{k}^{T} & q_{k+1}^{T} & e_{k+1}^{T} & f_{\Delta}^{T}
\end{array}\right]^{T} \\
y & =\left[\begin{array}{lll}
e_{k}^{T} & f_{k}^{T}
\end{array}\right]^{T}
\end{aligned}
$$

Using (17), (18a), (18b), (18c), and (18d), the ILC control problem can be stated using the following generalised plant:

$$
\left[\begin{array}{c}
q_{k} \\
q_{k+1} \\
e_{k+1} \\
f_{\Delta} \\
e_{k} \\
f_{k}
\end{array}\right]=\left[\begin{array}{ccccc}
0 & 0 & 0 & W_{o} & 0 \\
0 & 0 & 0 & 0 & W_{o} \\
W_{i} & -W_{i} & I & J & -J \\
0 & 0 & 0 & -R^{1 / 2} & R^{1 / 2} \\
0 & 0 & I & 0 & 0 \\
0 & 0 & 0 & I & 0
\end{array}\right]\left[\begin{array}{c}
p_{k} \\
p_{k+1} \\
e_{k} \\
f_{k} \\
f_{k+1}
\end{array}\right]
$$

We can now present the solution to the optimal ILC control problem.

Proposition 4.1: Given the minimax criterion (13) with cost functional (15) and generalised plant (19). Then, the ILC controller that solves (13) has the structure of (6) with learning filters:

$$
\begin{aligned}
\mathcal{Q} & =\left(J^{T} Q J+R+S\right)^{-1}\left(J^{T} Q J+R\right) \\
L & =\left(J^{T} Q J+R+S\right)^{-1} J^{T} Q,
\end{aligned}
$$

with:

$$
Q=\left(I-\frac{1}{2} \gamma^{-2} W_{i} W_{i}^{T}\right)^{-1}, \quad \text { and } \quad S=W_{o}^{T} W_{o} .
$$

Proof: The minimax game is solved by looking for a saddle point of (15), i.e., where its Jacobian equals zero. 
Substituting (19), with $w$ and $z$ according to (18a) and (18c), in (15) gives the following unconstrained cost functional:

$$
\begin{aligned}
\mathcal{J}=f_{k}^{T}\left(W_{o}^{T} W_{o}+R-\gamma^{2} I\right) f_{k} \\
-\gamma^{2}\left(p_{k}^{T} p_{k}+p_{k+1}^{T} p_{k+1}+e_{k}^{T} e_{k}\right) \\
\quad+f_{k+1}^{T}\left(W_{o}^{T} W_{o}+R\right) f_{k+1}-2 f_{k}^{T} R f_{k+1} \\
\quad+\left(W_{i} p_{k}-W_{i} p_{k+1}+e_{k}+J f_{k}-J f_{k+1}\right)^{T} \\
\quad\left(W_{i} p_{k}-W_{i} p_{k+1}+e_{k}+J f_{k}-J f_{k+1}\right) .
\end{aligned}
$$

The saddle point is achieved where the following partial derivatives equal zero:

$$
\begin{gathered}
\frac{\partial \mathcal{J}}{\partial p_{k}}=\left(W_{i}^{T} W_{i}-\gamma^{2} I\right) p_{k}-W_{i}^{T} W_{i} p_{k+1} \\
\quad+W_{i}^{T} e_{k}+W_{i}^{T} J f_{k}-W_{i}^{T} J f_{k+1}=0 \\
\frac{\partial \mathcal{J}}{\partial p_{k+1}}=\left(W_{i}^{T} W_{i}-\gamma^{2} I\right) p_{k+1}-W_{i}^{T} W_{i} p_{k} \\
\quad-W_{i}^{T} e_{k}-W_{i}^{T} J f_{k}+W_{i}^{T} J f_{k+1}=0 \\
\frac{\partial \mathcal{J}}{\partial f_{k+1}}=-J^{T} W_{i} p_{k}+J^{T} W_{i} p_{k+1}-\left(J^{T} J+R\right) f_{k} \\
-J^{T} e_{k}+\left(J^{T} J+R+W_{o}^{T} W_{o}\right) f_{k+1}=0 .
\end{gathered}
$$

Note that we do not take $\frac{\partial \mathcal{J}}{\partial e_{k}}$ and $\frac{\partial \mathcal{J}}{\partial f_{k}}$, since they are measured outputs and, therefore, given. Adding (23a) to (23b) yields that $p_{k+1}=-p_{k}$. Substituting this in (23a) gives us:

$p_{k}=\left(\gamma^{2}-2 W_{i}^{T} W_{i}\right)^{-1}\left(W_{i}^{T} e_{k}+W_{i}^{T} J f_{k}-W_{i}^{T} J f_{k+1}\right)$.

Finally, applying $p_{k}=-p_{k+1}$ to (23c), and substituting (24) herein, yields:

$$
\begin{gathered}
\left(J^{T}\left(I-\frac{1}{2} \gamma^{-2} W_{i} W_{i}^{T}\right)^{-1} J+R+W_{o}^{T} W_{o}\right) f_{k+1}= \\
J^{T}\left(I-\frac{1}{2} \gamma^{-2} W_{i} W_{i}^{T}\right)^{-1}\left(e_{k}+J f_{k}\right)+R f_{k},
\end{gathered}
$$

from which (20a) and (20b) can be obtained.

Note that the structure of this controller is equivalent to that of (10a) and (10b).

\section{RMC of the R-ILC Controller}

Like in $\mathcal{H}_{\infty}$ feedback control, the R-ILC controller is suboptimal, i.e., $\gamma>\gamma_{\text {opt }}$, where $\gamma_{\text {opt }}$ is the induced 2-norm of the closed loop system. The closed loop system $N$ is obtained by substituting $f_{k+1}=\mathcal{Q} f_{k}+L e_{k}$ into (19), and is given by:

$$
\left[\begin{array}{lll}
q_{k}^{T} & q_{k+1}^{T} & e_{k+1}^{T}
\end{array}\right]^{T}=N\left[\begin{array}{llll}
p_{k}^{T} & p_{k+1}^{T} & e_{k}^{T} & f_{k}^{T}
\end{array}\right]^{T},
$$

where:

$$
N=\left[\begin{array}{cccc}
0 & 0 & 0 & W_{o} \\
0 & 0 & W_{o} L & W_{o} \mathcal{Q} \\
W_{i} & -W_{i} & I-J L & J(I-\mathcal{Q})
\end{array}\right] .
$$

Note that we have taken $R=0$, since it does not contribute to RMC, and removed $f_{\Delta}$ from the closed loop system. The suboptimal controller can approach the optimal solution by iteratively lowering $\gamma$ for as long as $\gamma \geq\|N\|_{i 2}$ and the RMC condition of Proposition 3.1 is satisfied.

If for a given uncertainty model, no $\gamma$ can be found such that the R-ILC controller satisfies Proposition 3.1, a solution to the tuning of R-ILC can be found by observing that:

$$
W_{i} \Delta W_{o}=\beta^{-1} W_{i} \Delta \beta W_{o},
$$

i.e., by introducing a scaling factor $\beta$ in the uncertainty model. Although the $\mathrm{i} / \mathrm{o}$ behaviour of $W_{i} \Delta W_{o}$ does not change by defining $W_{i} \rightarrow \beta^{-1} W_{i}$ and $W_{o} \rightarrow \beta W_{o}$, the RILC controller has obtained an additional tuning parameter. Note that this $\beta$ can be interpreted as a $D$-scaling factor, as used in feedback $\mu$-synthesis [20].

Substitution of $W_{i} \rightarrow \beta^{-1} W_{i}$ and $W_{o} \rightarrow \beta W_{o}$ in the R-ILC controllers results in $Q$ and $S$

$$
Q=\beta^{2}\left(\beta^{2}-\frac{1}{2} \gamma^{-2} W_{i} W_{i}^{T}\right)^{-1}, \quad S=\beta^{2} W_{o}^{T} W_{o} .
$$

By dividing both $Q$ and $S$ by $\beta^{2}$, we find

$$
Q=\left(\beta^{2}-\frac{1}{2} \gamma^{-2} W_{i} W_{i}^{T}\right)^{-1}, \quad S=W_{o}^{T} W_{o} .
$$

Although for $W_{i}=W_{A}$ and $W_{o}=I$, no systematic tuning guidelines for $\gamma$ and $\beta$ in R-ILC have been found yet, for the case $W_{i}=I$ and $W_{o}=W_{A}$, we can exploit the fact that $Q$ is of the form $q^{-1} I$, with $q=\beta^{2}-\frac{1}{2} \gamma^{-2}$ for our tuning. With $Q=q^{-1} I$, the R-ILC controller becomes:

$$
\begin{aligned}
& L=\left(J^{T} J+q W_{o}^{T} W_{o}\right)^{-1} J^{T}, \\
& \mathcal{Q}=\left(J^{T} J+q W_{o}^{T} W_{o}\right)^{-1} J^{T} J .
\end{aligned}
$$

Tuning of the R-ILC controllers boils down to the iteratively lowering $q$ for as long as the appropriate RMC condition is satisfied. For a given value $q$, there always exists a $\beta$ and $\gamma_{\min }$ such that $\|N\|_{i 2}<\gamma_{\min }$ and $q=\beta^{2}-\frac{1}{2} \gamma_{\min }^{-2}$. Hence, after tuning $q$ there is no need to explicitly determine the $\gamma_{\min }$.

\section{Simulation Example}

In this Section, we illustrate the theory by means of a simulation example with an uncertain system. In this example, we compare the performance of the newly proposed RILC controller with a Linear Quadratic (LQ)-ILC controller, i.e., a norm optimal ILC controller with diagonal weighting matrices $Q, R$, and $S$.

\section{A. System Description}

For this example, we consider a model of the two-mass system used in [23]. The continuous time dynamics of this system are governed by the following transfer function:

$$
G(s)=\frac{d s+k}{m_{1} m_{2} s^{4}+\left(m_{1}+m_{2}\right) d s^{3}+\left(m_{1}+m_{2}\right) k s^{2}},
$$

where $m_{1}=2 \cdot 10^{-4}, m_{2}=1.6 \cdot 10^{-4}, d=5.66 \cdot 10^{-4}$, and $k=9.8$. Uncertainty is introduced by perturbing the values $d$ and $k$ between $95 \%$ and $105 \%$ of their nominal values. A discrete time equivalent of this model is obtained by using a 'zero-order-hold' approximation with a sampling 


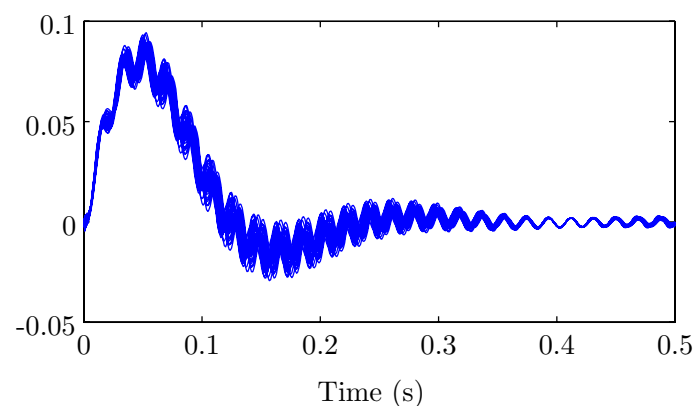

Fig. 3: The impulse response of the uncertain system $J_{p}$.

frequency of $1 \mathrm{kHz}$. Since this system is marginally stable, it is controlled using feedback with the following controller:

$$
K(s)=0.2 \frac{\left(\frac{1}{2 \pi \cdot 3} s+1\right)\left(\frac{1}{(2 \pi \cdot 52)^{2}} s^{2}+\frac{0.02}{2 \pi \cdot 52} s+1\right)}{\left(\frac{1}{2 \pi \cdot 20} s+1\right)\left(\frac{1}{(2 \pi \cdot 52)^{2}} s^{2}+\frac{2}{2 \pi \cdot 52} s+1\right)},
$$

which is implemented in discrete time using a Tustin approximation with a prewarp frequency of $52 \mathrm{~Hz}$. In case we use feedback control in conjunction with ILC, the process sensitivity is the relevant transfer function for ILC:

$$
J(z)=(I+G(z) K(z))^{-1} G(z) .
$$

The nominal system model is obtained by taking the nominal values for $k$ and $d$. The additive uncertainty bound of the process sensitivity is obtained by taking a Tustin approximation of the following continuous time bound:

$$
\begin{aligned}
& W_{A}(s)=5 \cdot 10^{-6} \cdot \frac{\left(\frac{1}{(2 \pi \cdot 0.2)^{2}} s^{2}+\frac{2}{2 \pi \cdot 0.2} s+1\right)}{\left(\frac{1}{(2 \pi \cdot 5.2)^{2}} s^{2}+\frac{0.6}{2 \pi \cdot 5.2} s+1\right)} . \\
& \frac{\left(\frac{1}{(2 \pi \cdot 51)^{2}} s^{2}+\frac{1.1}{2 \pi \cdot 51} s+1\right)\left(\frac{1}{(2 \pi \cdot 54.5)^{2}} s^{2}+\frac{1.1}{(2 \pi \cdot 54.5)} s+1\right)}{\left(\frac{1}{(2 \pi \cdot 51)^{2}} s^{2}+\frac{0.04}{2 \pi \cdot 51} s+1\right)\left(\frac{1}{(2 \pi \cdot 54.5)^{2}} s^{2}+\frac{0.042}{(2 \pi \cdot 54.5)} s+1\right)} .
\end{aligned}
$$

The lifted system description of (4) is obtained by defining $J, W_{i}$ and $W_{o}$ as given in (3). The perturbed system's impulse response and the defined trajectory for ILC (which is in fact the reference trajectory filtered by the sensitivity function $\left.(I+G(z) K(z))^{-1}\right)$ are depicted in Fig. 3 and Fig. 4 , respectively.

\section{B. RMC of a R-ILC Controlled System}

With the system described, we can design the ILC controllers, such that the ILC algorithm is RMC.

For the LQ-optimal controller, Proposition 3.1 gives a sufficient condition for RMC. The ILC controller with learning filters (10a), (10b), with $Q=I, R=0$, and $S=0.7 \cdot I$, guarantees RMC.

For the R-ILC controller, we represent our uncertainty by choosing $W_{i}=I$ and $W_{o}=W_{A}$. With this choice of uncertainty, no $\gamma$ can be found such that the R-ILC controller satisfies the conditions of Proposition 3.1. We therefore introduce the $\beta$-factor as argued in Section IV-C

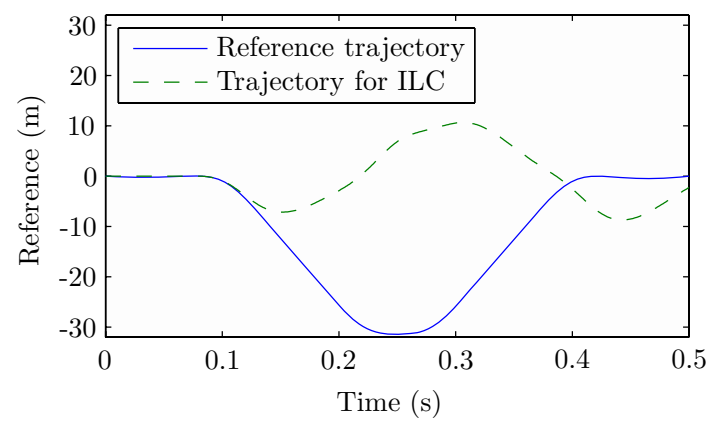

Fig. 4: The applied reference trajectory $r$.

to achieve RMC. Then, tuning the controller boils down to choosing $q$. It turns out that choosing $q=1250$ makes the R-ILC controller RMC.

Fig. 5 shows that in both situations the 2-norm of the command signal converges monotonically, and Fig. 6 depicts the 2-norm of the error for both ILC controllers. These results are based on simulations with 25 samples of $J_{p} \in \Pi$. It can be concluded that both controllers achieve RMC, however the R-ILC has a converged error whose norm is approximately 10 times smaller than that of the LQ-ILC controller. The nonzero asymptotic value of $\left\|f_{k}-f_{\infty}\right\|_{2}$ is due to numerical errors.

It can be reasoned why the R-ILC controller outperforms the LQ-ILC controllers by considering the power spectral density of the error at trial $k=10$, see Fig. 7. In LQ-ILC, the $\mathcal{Q}$-filter has a low pass characteristic that cuts off all singular values smaller than a certain threshold [8]. Because the uncertainty of our example is associated with large singular values, the cut off value of the $\mathcal{Q}$-filter is relatively high. The $\mathcal{Q}$-filter of the R-ILC controller, however, cuts off singular values that are associated with singular vectors that are uncertain, independent of the magnitude of the singular value itself. As a result, R-ILC only gives robustness where it is required.

\section{CONCLUSIONS}

In this paper, we have presented a novel Iterative Learning Control (ILC) strategy that is robust against model

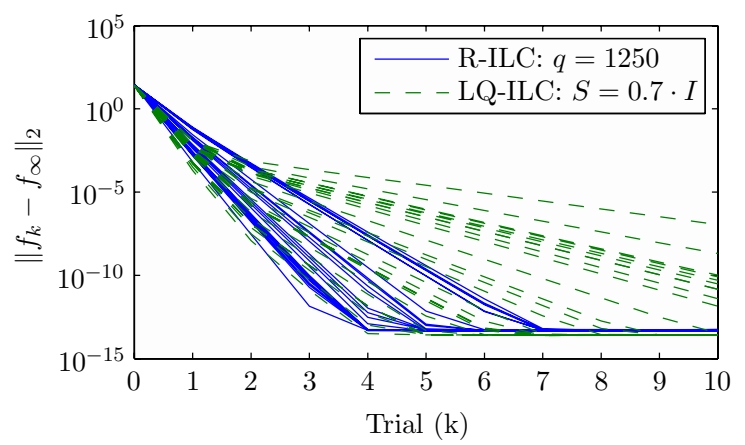

Fig. 5: Convergence of the command signal for both the RILC and the LQ-ILC controller. 


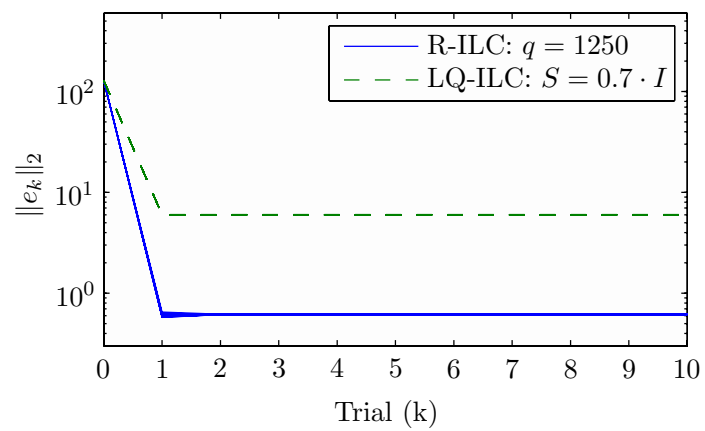

Fig. 6: Convergence of the error for both the R-ILC and the LQ-ILC controller.

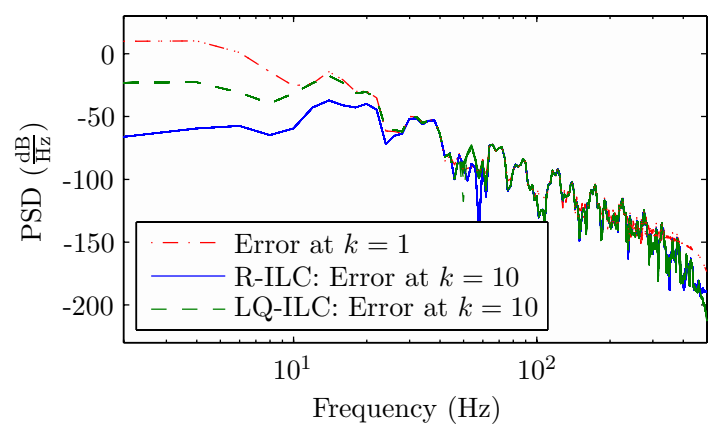

Fig. 7: Power spectral density of the error.

uncertainty, specified by a nominal model and an additive uncertainty bound. The resulting controller is the result of an optimisation over an induced norm, and acts on a finite-time interval, exploits noncausal behaviour, and incorporates an uncertainty model. An example has shown that the presented robust ILC controller can outperform linear quadratic ILC controllers, in terms of performance loss that is necessarily sacrificed to obtain the required amount of robustness.

\section{REFERENCES}

[1] H. Ahn, K. Moore, and Y. Chen. Monotonic convergent iterative learning controller design based on interval model conversion. IEEE Trans. Autom. Control, 51(2):366-371, 2006.

[2] H. Ahn, K. Moore, and Y. Chen. Schur stability radius bounds for robust iterative learning controller design. In Proc. of the American Control Conf., pages 178-183, Portland, Orlando, FL, USA, June 2005.

[3] N. Amann, D. Owens, and E. Rogers. Iterative learning control for discrete time systems using optimal feedback and feedforward actions. In Proc. of the 34th Conf. on Decision and Control, pages 1696-1701, New Orleans, LA, USA, Deember 1995.

[4] N. Amann, D. Owens, E. Rogers, and A. Wahl. An $\mathcal{H}_{\infty}$ approach to linear iterative learning control. Int. Journal of Adaptive Systems and Signal Processing, 10:767-781, 1996.

[5] T. Başar and P. Bernhard. $\mathcal{H}_{\infty}$ Optimal Control and Related Minimax Design Problems - A Dynamic Game Approach. Birkhauser, Boston, MA, USA, 1991.

[6] S. Boyd and L. Vandenberghe. Convex Optimization. Cambridge University Press, 2004.

[7] D. Bristow, M. Tharayil, and A. Alleyne. A survey of iterative learning control; a learning-based method for high-performance tracking control. IEEE Control Systems Magazine, 26(3):96-114, 2006.

[8] B. Dijkstra and O. Bosgra. Noise suppression in buffer-state iterative learning control, applied to a high precision wafer-stage. In Proceedings of the 2002 IEEE International Conference on Control
Applications, pages 998-1003, Glasgow, Scotland, UK, September 2002.

[9] T. Donkers, J. van de Wijdeven, and O. Bosgra. Robustness against model uncertainties of norm optimal iterative learning control. In Submitted for Proc. of the American Control Conf., 2008.

[10] J. Frueh and M. Phan. Linear quadratic optimal learning control. Int. Journal of Control, 73(10):832-839, 2000.

[11] J. Ghosh and B. Paden. Pseudo-inverse based iterative learning control for plant with unmodelled dynamics. In Proc. of the American Control Conf., pages 472-476, Chicago, IL, USA, June 2000.

[12] P. Goldsmith. On the equivalence of causal LTI iterative learning control and feedback control. Automatica, 38(4):703-708, 2002.

[13] D. Gorinevsky. Loop shaping for iterative learning control of batch processes. IEEE Control Systems Magazine, 22(6):55-65, 2002.

[14] S. Gunnarsson and M. Norrlöf. On the design of ILC algorithms using optimisation. Automatica, 37(12):2011-2016, 2001.

[15] D. Limebeer, M.Green, and D. Walker. Discrete time $\mathcal{H}_{\infty}$ control. In Proc. of the 28th Conf. on Decision and Control, pages 392-396, Tampa, FL, USA, December 1989.

[16] K. Moore, Y. Chen, and H. Ahn. Algebraic $\mathcal{H}_{\infty}$ desing of higher order iterative learning controllers. In Proc. of the 2005 IEEE Int. Symposium on Intelligent Control, pages 1213-1218, Limassol, Cyprus, June 2005.

[17] M. Norrlöf and S. Gunnarsson. Time and frequency convergence properties in iterative learning control. Int. Journal of Control, 75(14):1114-1126, 2002.

[18] M. Phan and R. Longman. A mathematical theory of learning control for linear discrete multivariable systems. In Proc. of the AIAA/AAS Astrodynamics Conf., pages 740-746, Minneapolis, MN, USA, August 1988.

[19] D. de Roover and O. Bosgra. Synthesis of a robust multivariable iterative learning controllers with application to a wafer stage motion system. Int. Journal of Control, 73(10):968-979, 2000.

[20] S. Skogestad and I. Postlethwaite. Multivariable Feedback Control. John Wiley \& Sons, Ltd., 2005.

[21] M. Verwoerd, G. Meinsma, and T. de Vries. On the use of noncausal LTI operators in iterative learning control. In Proc. of the 41th Conf. on Decision and Control, pages 3362-3366, Las Vegas, NV, USA, December 2002.

[22] J. van de Wijdeven and O. Bosgra. Noncausal finite-time robust Iterative Learning Ccontrol. In To appear in Proc. of the 46th Conf. on Decision and Control, New Orleans, LA, USA, 2007.

[23] J. van de Wijdeven and O. Bosgra. Residual vibration suppression using Hankel iterative learning control. Int. Journal of Robust and Nonlinear Control, 2007. 